\title{
Combined PET and MRI for the masses!
}

\section{At least for the cardiac ones}

\author{
Christoph Rischpler, ${ }^{\mathrm{a}}$ and Robert Seifert ${ }^{\mathrm{a}}$ \\ a Department of Nuclear Medicine, University Hospital Essen, University of Duisburg-Essen, \\ Essen, Germany
}

Received Oct 29, 2021; accepted Oct 31, 2021

doi: $10.1007 / \mathrm{s} 12350-021-02881-7$

See related article, pp. 1504-1517

About a decade ago, the dream of many nuclear physicians, radiologists, and even engineers came true and the first fully integrated, commercially available PET/MRI device was launched, leading to a large body of work investigating the synergy of these two imaging modalities. ${ }^{1}$ It did not take long for promising results to be published in oncological $^{2}$ and also neurological fields of application. ${ }^{3}$ In cardiology, on the other hand, the integrated analysis of PET and MRI was initially mainly employed in research projects, either in clinical studies ${ }^{4,5}$ or case reports, ${ }^{6,7}$ albeit relatively numerous. The first real convincing clinical application was published in this very journal: a study was able to show the benefit of hybrid FDG PET/MRI in myocarditis patients. ${ }^{8}$ Since then, other clinically significant cardiac applications of combined PET and MRI imaging have been published. One that is particularly worth highlighting is a study of sarcoidosis patients with the clinical question of cardiac involvement. ${ }^{9,10}$ Here, combined PET and MR imaging has demonstrated its added value primarily by showing greater accuracy with combined analyses. Another very significant population of patients in whom added value of PET/MRI has been suggested is patients with cardiac tumors. It is understandable that in these patients, the most accurate imaging is highly desirable to avoid the frequent need for diagnosis by biopsy. Although the majority of myocardial tumors, approximately three-quarters, are benign, misdiagnosis can of

Reprint requests: Christoph Rischpler, Department of Nuclear Medicine, University Hospital Essen, University of Duisburg-Essen, Essen, Germany; christoph.rischpler@uk-essen.de

J Nucl Cardiol 2022;29:1518-9.

$1071-3581 / \$ 34.00$

Copyright (c) 2021 The Author(s) course have fatal consequences. On the other hand, due to the high complication rate of the procedure, an unnecessary biopsy in a benign myocardial mass can also result in high morbidity and rarely even lethality. While there was a pilot study back in 2015 that looked at the value of integrated analysis of PET and MRI in the study of cardiac masses, the major shortcoming of that study was the small case number of 20 patients. ${ }^{11}$ Another study that was limited to FDG PET/CT came up with only 24 patients as well. ${ }^{12}$ However, in the highly interesting study by Aghayev et al published in the current issue of the JNC, more than three times as many patients were examined, resulting in a cohort of 72 patients. ${ }^{13}$ Furthermore, histological correlation was present in the majority of patients. Last but not least, the patients were followed-up and thus high-risk features regarding outcome could be investigated. In the current study, MRI was shown to achieve high sensitivity and specificity in differentiating benign vs. malignant cardiac tumors if 4 of 9 MRI features were positive (AUC 0.91). Individual MRI features showed significantly lower accuracy, with, interestingly, mere lesion size showing the highest accuracy. The inclined reader (particularly nuclear medicine physicians) may take some pride in the fact that a single, simple feature of PET, namely an $\mathrm{SUV}_{\max } / \mathrm{blood}$ pool ratio $>3$, showed similar accuracy compared with the more complex MRI analysis (AUC 0.87). In the end, the integrated analysis of PET and MRI did not show a higher accuracy, but the authors could prove the added value in single complex cases. Outcome analysis also demonstrated that both MRI, by showing an infiltrative border, and PET, by showing focal extracardiac FDG uptake, may have prognostic significance. As promising as the results are, further questions remain unanswered: the PET analysis in the present study was relatively simplistic compared with the MRI analysis (maximal uptake in FDG PET vs 9 different features in MRI), so the question remains whether a more elaborate analysis of PET, e.g., using dynamic acquisition or additional parameters such as $\mathrm{SUV}_{\text {peak }}$, 
Table 1. Comparison of the diagnostic performance of FDG PET vs. MRI in cardiac masses

\begin{tabular}{lllll}
\hline Study & No. of patients & FDG PET & \multicolumn{1}{c}{ MRI } & FDG PET + MRI \\
\hline Rahbar et al. ${ }^{12}$ & 24 & $100 \% / 86 \%$ & - & - \\
Nensa et al. ${ }^{11}$ & 20 & $100 \% / 92 \%$ & $100 \% / 92 \%$ & $100 \% / 100 \%$ \\
Aghayev et al. ${ }^{13}$ & 72 & $85 \% / 88 \%$ & $98 \% / 84 \%$ & $98 \% / 84 \%$
\end{tabular}

Given percentage values are sensitivity/specificity

$\mathrm{SUV}_{\text {mean }}$, radiomics of PET, metabolic tumor volume, can further increase diagnostic accuracy (Table 1).

Nevertheless, the study by Aghayev et al has shown one thing: an integrated PET and MRI analysis represents a promising diagnostic tool of cardiac masses and has the potential to become the clinical standard for this question. We therefore advocate "PET and MRI for cardiac masses!",

\section{Funding} $D E A L$.

Open Access funding enabled and organized by Projekt

\section{Disclosures}

For this work, Dr. Seifert was partly supported by the University Medicine Essen Clinician Scientist Academy (UMEA). Dr. Rischpler reports a research grant from Pfizer, consultancy for Adacap and Pfizer, speaker honoraria from Adacap, Alnylam, BTG, GE Healthcare, Pfizer and Siemens Healthineers.

\section{Open Access}

This article is licensed under a Creative Commons Attribution 4.0 International License, which permits use, sharing, adaptation, distribution and reproduction in any medium or format, as long as you give appropriate credit to the original author(s) and the source, provide a link to the Creative Commons licence, and indicate if changes were made. The images or other third party material in this article are included in the article's Creative Commons licence, unless indicated otherwise in a credit line to the material. If material is not included in the article's Creative Commons licence and your intended use is not permitted by statutory regulation or exceeds the permitted use, you will need to obtain permission directly from the copyright holder. To view a copy of this licence, visit http://creativecommons.org/licenses/by/4.0/.

\section{References}

1. Delso G, Fürst $\mathrm{S}$, Jakoby B, et al. Performance measurements of the Siemens mMR integrated whole-body PET/MR scanner. J Nucl Med 2011;52:1914-22.
2. Drzezga A, Souvatzoglou M, Eiber M, et al. First clinical experience with integrated whole-body PET/MR: Comparison to PET/ CT in patients with oncologic diagnoses. J Nucl Med 2012;53:84555.

3. Hitz S, Habekost C, Fürst S, et al. Systematic comparison of the performance of integrated whole-body PET/MR imaging to conventional PET/CT for 18F-FDG brain imaging in patients examined for suspected dementia. J Nucl Med 2014;55:923-31.

4. Rischpler C, Langwieser N, Souvatzoglou M, et al. PET/MRI early after myocardial infarction: Evaluation of viability with late gadolinium enhancement transmurality vs. 18F-FDG uptake. Eur Heart J Cardiovasc Imaging 2015;16:661-9.

5. Rischpler C, Dirschinger RJ, Nekolla SG, et al. Prospective evaluation of $18 \mathrm{~F}$-fluorodeoxyglucose uptake in postischemic myocardium by simultaneous positron emission tomography/magnetic resonance imaging as a prognostic marker of functional outcome. Circ Cardiovasc Imaging 2016;9:e004316.

6. Rischpler C, Nekolla SG, Kossmann H, et al. Upregulated myocardial CXCR4-expression after myocardial infarction assessed by simultaneous GA-68 pentixafor PET/MRI. J Nucl Cardiol 2016;23:131-3.

7. Goedel A, Hoellein A, Rischpler C, Götze K. B-lymphoblastic lymphoma: A heartening diagnosis. Eur Heart $\mathrm{J}$ Cardiovasc Imaging 2015;16:116.

8. Nensa F, Kloth J, Tezgah E, et al. Feasibility of FDG-PET in myocarditis: Comparison to CMR using integrated PET/MRI. J Nucl Cardiol 2018;25:785-94.

9. Vita T, Okada DR, Veillet-Chowdhury M, et al. Complementary value of cardiac magnetic resonance imaging and positron emission tomography/computed tomography in the assessment of cardiac sarcoidosis. Circ Cardiovasc Imaging 2018;11:e007030.

10. Dweck MR, Abgral R, Trivieri MG, et al. Hybrid magnetic resonance imaging and positron emission tomography with fluorodeoxyglucose to diagnose active cardiac sarcoidosis. JACC Cardiovasc Imaging 2018;11:94-107.

11. Nensa F, Tezgah E, Poeppel TD, et al. Integrated 18F-FDG PET/ MR imaging in the assessment of cardiac masses: A pilot study. $\mathbf{J}$ Nucl Med 2015;56:255-60.

12. Rahbar K, Seifarth $H$, Schäfers $M$, et al. Differentiation of malignant and benign cardiac tumors using 18F-FDG PET/CT. J Nucl Med 2012;53:856-63.

13 Aghayev A, Cheezum MK, Steigner ML, et al. Multimodality imaging to distinguish between benign and malignant cardiac masses. J Nucl Cardiol 2021. https://doi.org/10.1007/s12350-02102790-9.

Publisher's Note Springer Nature remains neutral with regard to jurisdictional claims in published maps and institutional affiliations. 